Provided for non-commercial research and education use. Not for reproduction, distribution or commercial use.

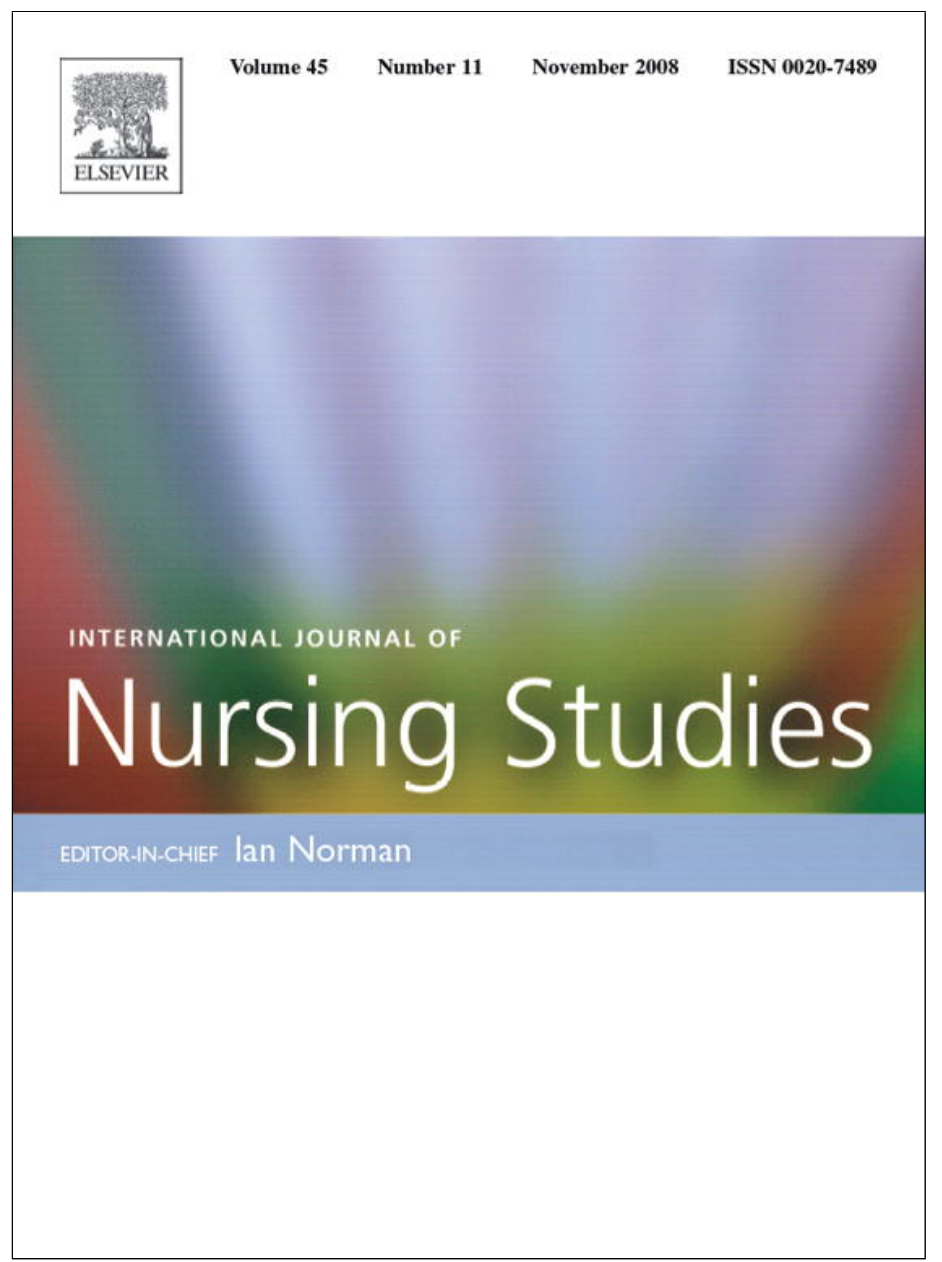

This article appeared in a journal published by Elsevier. The attached copy is furnished to the author for internal non-commercial research and education use, including for instruction at the authors institution and sharing with colleagues.

Other uses, including reproduction and distribution, or selling or licensing copies, or posting to personal, institutional or third party websites are prohibited.

In most cases authors are permitted to post their version of the article (e.g. in Word or Tex form) to their personal website or institutional repository. Authors requiring further information regarding Elsevier's archiving and manuscript policies are encouraged to visit:

http://www.elsevier.com/copyright 


\title{
Mothers' attitudes towards using services preventing mother-to-child HIV/AIDS transmission in Zimbabwe: An interview survey
}

\author{
Clara Chivonivoni $^{a}$, Valerie J. Ehlers ${ }^{b}{ }^{*}$, Janetta H. Roos ${ }^{b}$ \\ ${ }^{\text {a } U n i t e d ~ B u l a w a y o ~ H o s p i t a l s, ~ B u l a w a y o, ~ Z i m b a b w e ~}$ \\ ${ }^{\mathrm{b}}$ Department of Health Studies, University of South Africa, PO Box 392, Pretoria. 0003, South Africa
}

Received 28 June 2007; received in revised form 31 March 2008; accepted 1 April 2008

\begin{abstract}
Background: In developing countries vertical (mother-to-child) transmission of HIV/AIDS is responsible for 5-10\% of all new HIV infections. HIV positive mothers can transmit HIV to their babies during pregnancy, childbirth and breast-feeding. Antiretroviral drugs are effective in reducing the risk of vertical transmission of HIV/AIDS.

Aim: The main focus was to describe mothers' attitudes towards using services for preventing vertical transmission of HIV/ AIDS.

Design: A non-experimental, descriptive design with a survey approach was used.

Setting: The study was conducted at one hospital in Bulawayo, Zimbabwe, that offers both prenatal clinic and maternity, including prevention of vertical transmission, services.

Participants: Fifty pregnant women, who attended prenatal clinics in Bulawayo and who booked to deliver their babies in the hospital's maternity section, were interviewed.

Method: A structured interview survey was used to collect data.

Results: The interviewed women required more knowledge about preventing vertical transmission of HIV/AIDS. Many pregnant women would not use the services available for the prevention of vertical transmission of HIV/AIDS, for personal, financial and cultural reasons. However, the most important barriers preventing pregnant women from using free prevention of vertical transmission services were structural ones. Only pregnant women who attended prenatal clinics and delivered their babies in hospital could access these services. Prenatal and delivery services might be beyond the financial reach of many Zimbabwean women, making prevention of vertical transmission services inaccessible to them. Free infant formula could not be accessed at hospitals and clinics because of transport costs.
\end{abstract}

(C) 2008 Elsevier Ltd. All rights reserved.

Keywords: Anti-retroviral drugs; HIV/AIDS; Prevention of vertical transmission of HIV/AIDS; Voluntary counseling and testing; Zimbabwe

Abbreviations: AIDS, auto-immune deficiency syndrome; ARV, anti-retroviral (drugs); HIV, human immune deficiency virus; MOHCW, Ministry of Health and Child Welfare (of Zimbabwe).

* Corresponding author. Tel.: +27 12347 8287; fax: +27 123478287 .

E-mail address: ehlersjh@mweb.co.za (V.J. Ehlers).
What is already known about the topic?

- Vertical transmission of HIV/AIDS can be prevented by administering anti-retroviral drugs to the HIV positive mother and the newborn infant and by providing formula feeds for the infant. 
- Nevirapine and infant formula are available in Zimbabwe but many pregnant women fail to use these services.

\section{What this paper adds?}

- Women lack knowledge about the prevention of vertical transmission anti-retroviral drugs and formula feeds to protect their newborn children from vertical transmission of HIV/AIDS in Zimbabwe.

- Even the women who had such knowledge would not consent to voluntary counselling and testing, could not access caesarian sections and might breastfeed their babies because using the free infant formula feeds would entail transport costs.

- Policy implications are that prenatal and delivery services should be free of charge, free infant formula should be distributed throughout communities, women who do not deliver their babies in hospitals should also have access to services preventing the vertical transmission of HIV/ AIDS.

\section{Introduction}

"In 1998 it was estimated that HIV/AIDS kills approximately two million people in sub-Sahara Africa" (Southern Africa HIV/AIDS Action, 2000, p. 2). An estimated 570,000 children, aged 14 years or younger, most of them from the sub Sahara Africa region became HIV positive in 1999, many from vertical transmission (Bang, 2000). In developing countries, vertical transmission is responsible for 5-10\% of all new HIV infections. Of the children born to HIV positive mothers, between $33.3 \%$ and $50.0 \%$ is HIV positive. Mothers can transmit HIV/AIDS to their babies during pregnancy, childbirth and breastfeeding.

\subsection{Purpose and objectives}

Zimbabwe has one of the world's highest HIV infection rates. This translates to an estimated 50,000 infants being infected annually (MOHCW, 2002). Lowering the incidence of vertical transmissions involves voluntary counseling and testing for HIV/AIDS; the use of anti-retroviral drugs; counseling and support for safe infant feeding; performing caesarian sections to reduce the chances of vertical transmission during the birth process; follow-up visits of the HIV positive mothers, their children and their families (MOHCW, 2002).

During 2005 the first three services were available free of charge at the participating prenatal clinic, and/or its associated hospital, in Bulawayo. A single dose of $200 \mathrm{mg}$ Nevirapine is administered to a woman in established labour. The baby is given Nevirapine as a single dose of $2 \mathrm{mg} / \mathrm{kg}$ body weight within $72 \mathrm{~h}$ of birth. These HIV positive mothers (MOHCW, 2002) receive consistent supplies of infant formula feeds. Only pregnant women who are HIV positive, and who delivered their babies in hospitals, can access these free services. However, even accessing these free services pose huge challenges to the women concerned. Zimbabwe faces food shortages with empty shop shelves, fuel shortages characterized by empty cars queued up for miles outside empty petrol stations, virtually no public transport, the highest inflation rate in the world exceeding $1000 \%$ per month and these hardships are aggravated by joblessness and political unrest. Zimbabwean women's challenges for daily survival make it hard for them to invest some of their scarce resources to prevent vertical transmission of HIV/AIDS.

The purpose of this study was to identify women's attitudes towards using services for preventing vertical transmission of HIV/AIDS in Bulawayo. The objectives of the study were to identify pregnant women's attitudes, knowledge and practices towards using services for the prevention of vertical transmission of HIV/AIDS and to make recommendations for improved utilization of these services in Bulawayo.

\subsection{Significance of the research}

From January till October 2005, the participating hospital's prenatal statistics reveal that out of 2647 women, only $693(26.18 \%)$ were tested for HIV, and 152 (21.93\%) were HIV positive (Chivonivoni, 2006). These figures imply that $1954(73.07 \%)$ of the 2674 pregnant women who attended this clinic were not tested for HIV and could thus not use the free services for preventing vertical transmission of HIV/ AIDS.

The results of the study may inform and support clinical decisions for motivating more pregnant women to make use of voluntary HIV/AIDS counseling and testing services, the use of Nevirapine and formula feeds to reduce the risk of vertical transmission of HIV/AIDS. If more women in the Bulawayo region would use the available prevention of vertical transmission of HIV/AIDS services, then the number of babies suffering and/or dying from HIV/AIDS in this area could decline.

\subsection{Selected theoretical framework}

The effects of people's beliefs on their health actions were formulated as the Health Belief Model by Becker (1974), based on work done for the public health services in the United States of America during the 1960s.

The study was based on Onega's (2000) description of the Health Belief Model, comprising four major tenets (individual perceptions, modifying factors, barriers to implementing actions, and the likelihood of implementing and sustaining recommended actions) applied to the prevention of vertical transmission of HIV/AIDS.

Individual perceptions of pregnant women, in relation to the prevention of vertical transmission of HIV/AIDS, such as believing that HIV is irrelevant and/or harmless, could influence them not to get tested for HIV and/or not to use the available services. 
Modifying factors include demographic aspects (such as the mothers' ages, marital status, gender, educational status and cultural beliefs); social psychological issues, social values, beliefs and practices of antenatal mothers in relation to the prevention of vertical transmission of HIV/AIDS, including cultural and economic factors.

Barriers to implementing effective health actions include encountering problems in accessing prenatal services, voluntary counseling and testing services, Nevirapine (and/or other anti-retroviral drugs), formula feeds and possibly health care workers' negative attitudes.

The likelihood of taking recommended preventive health actions (using prevention of vertical transmission services), depends on the specific benefits expected (preventing vertical transmission) counterbalanced by the effort required (accessing the service, incurring costs, coping with fear and possible stigma) from the health action(s). In Zimbabwe, culture and demography intersect to modify women's individual perceptions and barriers influencing their implementation of preventive health actions such as bottle-feeding their babies. Women require time, energy and money to get their babies' formula from hospitals/clinics, to find clean water and to boil the water-a formidable task with severe fuel shortages, and extended interruptions in electricity supplies for those who can afford electricity. Women who do not breastfeed their babies in Zimbabwe are stigmatized to the extent that their husbands/partners might abandon them and their babies.

\section{Methods}

The study used a non-experimental quantitative survey, which was exploratory and descriptive in nature, because limited literature was available about the attitudes, knowledge and practices of prenatal mothers in Bulawayo to prevent vertical transmission of HIV/AIDS. A structured interview schedule was developed, based on the available international literature, Zimbabwe's Ministry of Health's guidelines and World Health Organization documents.

\subsection{Research setting}

The research was conducted at one hospital in Bulawayo that offered prenatal, delivery and prevention of vertical transmission services. As Zimbabwe suffered extreme fuel shortages during the data collection phase (1 August to 30 September 2005), it was difficult and expensive to travel to different health care facilities.

\subsection{Research population and sample}

The accessible population comprised all the women who attended the prenatal clinic and who booked their deliveries at the participating Bulawayo hospital, as reflected on the prenatal clinic's list. Thus women comprising the accessible population had to be pregnant, attend the prenatal clinic, have booked to deliver their babies in the hospital, and be willing to be interviewed about the prevention of vertical transmission (women who attended the prenatal clinic but who opted for home deliveries would not have access to prevention of vertical transmission services, and could not participate in this study).

During every pregnant woman's visit to the prenatal clinic, the purpose of the study was explained to her and her voluntary participation was requested. The sample size was not pre-determined, but as many interviews as possible were conducted during the data collection phase, from 1 August till 30 September 2005. Pregnant women faced high transport costs (due to inflationary fuel prices and severe fuel shortages in Zimbabwe) that limited their visits to the prenatal clinic. Fifty interviews were conducted during the data collection phase.

\subsection{The data collection instrument}

A structured interview schedule was used to collect data from the 50 participating pregnant women. Interviewers who use structured interview schedules ask the same questions in the same order and in the same manner (Brink, 1999). The interviewer asked questions concerning interviewees' biographic information (Section A); knowledge about the prevention of vertical transmission of HIV/AIDS (Section B); attitudes towards the prevention of vertical transmission of HIV/AIDS, including the preferred mode of delivery, type of infant feeding and the use of anti-retroviral drugs (Section C); and their practices to prevent vertical transmission of HIV/AIDS (Section D).

\subsubsection{Reliability and validity of the structured interview schedule}

"Reliability is the consistency with which the instrument measures an attribute" (Polit and Hungler, 1999, p. 255). This implies that the less variation a research instrument shows in repeated applications, under comparable conditions, the higher its reliability. The structured interview schedule was pre-tested by interviewing five pregnant women who were excluded from participation in the actual study. These women encountered no problems in understanding the questions and required 30-40 min to answer all the items. No discrepancies were detected between data obtained during the pre-test and the actual data collected from 50 women.

Two doctors and six professional nurses, who administer Nevirapine, scrutinized the interview schedule's items. Two nurse researchers and a statistician from the University of Bulawayo also evaluated the relevance of the items contained in the structured interview schedule. Thus eleven practitioners in the field of HIV/AIDS and/or health research evaluated the face validity of the structured interview schedule's items. Their suggestions involved simplifying some questions, and rephrasing others to become two separate items. 
The external validity might have been compromised by using a convenience sample of 50 pregnant women attending one prenatal clinic. This implies that the research results cannot be generalized to other sites.

\subsection{Data collection procedure}

The first author speaks English, Ndebele and Shona (the languages spoken mostly in the Bulawayo area of Zimbabwe) and conducted all the interviews. All the interviewed pregnant women understood and spoke English.

Every pregnant woman who visited the prenatal clinic during the data collection phase, from 1 August till 30 September 2005, was privately informed about the purpose of the study. Each woman was informed that she had the sole power to decide whether or not to be interviewed without incurring any ill effects whatsoever. No signed consent was requested but verbal consent was accepted to ensure anonymity. All interviewed women were older than 21. Every interviewee could refrain from answering specific questions should she wish to do so and she could withdraw from participation at any stage during the interview. Only the interviewer and the statistician had access to the completed interview schedules. After the publication of the research report, these documents were destroyed.

\subsection{Data analysis}

The Microsoft Excel 2000 computer program was used to categorize and analyze data from the completed structured interview schedules and to generate descriptive statistics.

\section{Results}

The results will be presented according to the major components of the Health Belief Model, namely individual perceptions, modifying factors, barriers encountered in implementing health actions, and factors influencing the likelihood of taking recommended health actions.

\subsection{Individual perceptions about HIV/AIDS and the prevention of vertical transmission of HIV/AIDS}

Out of the 50 interviewees, $62.0 \%(n=31)$ knew what prevention of vertical transmission of HIV/AIDS meant. Of these 31 women, $64.5 \%(n=19)$ obtained this knowledge from the media while $35.5 \%(n=11)$ obtained it from health centers. Only $28(56.0 \%)$ women knew that breastfeeding increased the risk of transmitting HIV to the baby.

\subsection{Modifying factors}

The major modifying factors related to the interviewed women's demographics. The majority of the interviewees $(80.0 \% ; n=40)$ were 30 years old or younger; pregnant for the first or second time $(72.0 \%: n=36)$ and were married $(88.0 \% ; n=44)$. Only $9(18.0 \%)$ women had completed primary school while $41(82.0 \%)$ had reached secondary or tertiary education levels, indicating that the majority should be able to comprehend the prevention of vertical transmission of HIV/AIDS. In spite of their relatively high levels of education only $15(30.0 \%)$ women were employed.

\subsection{Barriers to implementing effective health actions to prevent vertical transmission of HIV/AIDS}

The identified barriers included communication problems with husbands/partners, breastfeeding traditions and the mode of delivery.

\subsubsection{Communication with husbands/partners about preventive vertical transmission actions}

Although only $27(54.0 \%)$ of the interviewed women were willing to be tested for HIV, 31 (62.0\%) had discussed the prevention of vertical transmission with their husbands/ partners. The $19(38.0 \%)$ women who failed to do so feared that their husbands/partners might not want to engage in sexrelated discussions $(n=8 ; 42.1 \%)$, might label the woman as having "loose morals" $(n=6 ; 31.6 \%)$ or react violently towards discussions of HIV/AIDS topics $(n=5 ; 26.3 \%)$.

\subsubsection{Breastfeeding}

Out of the 50 interviewed women, only 28 (56.0\%) knew that breastfeeding increased the risk of vertical transmission of HIV/AIDS. As many as $22(44.0 \%)$ of the women would breast feed their babies because "breast milk is best for babies" and 12 out of these $22(54.5 \%)$ women indicated that they had to breast feed their babies as they were too poor to pay for transport to get their babies' free formula feeds from clinics.

\subsubsection{Mode of delivery}

If found to be HIV positive, 54\% $(n=27)$ of the interviewed women would prefer to deliver their babies by caesarian section to minimise the risk of HIV transmission during the birth process.

\subsection{Likelihood of taking recommended health actions}

The pregnant women's likelihood of taking recommended health actions to prevent vertical transmission of HIV/AIDS depended on their willingness to be tested for HIV. Unless the pregnant woman's HIV status is known no preventive action can be taken. Only $27(54.0 \%)$ of these pregnant women wanted to be tested for HIV, while 14 $(28.0 \%)$ were unwilling to be tested and $9(18.0 \%)$ were unsure.

Out of the 27 women willing to be tested for HIV, 26 (96.3\%) wanted to know their results. As many as 23 (86.2\%) would tell someone if they were HIV positive; $18(78.3 \%)$ would tell their husbands/partners while 5 
(21.7\%) would tell their siblings and/or parents. Of those who would tell the husbands/partners, $39.0 \%$ would do so in order to commence taking anti-retroviral drugs, $33.0 \%$ for using condoms and $28.0 \%$ for getting financial support from their husbands/partners.

\section{Discussion}

\subsection{Individual perceptions}

All interviewed women attended prenatal clinics. Consequently health education about HIV/AIDS and the prevention of vertical transmission should have been received by all of them, not only by $35.5 \%$ as indicated by these results. All pregnant women should know that breastfeeding increases the likelihood of transmitting HIV, a fact reportedly unknown to $44.0 \%$ of the interviewed pregnant women. More women gained knowledge about the prevention of vertical transmission from the media than from the health facilities in Bulawayo. The participating prenatal clinic in Bulawayo, attended by all the interviewed women, was not their primary source of information about the prevention of vertical transmission.

\subsection{Modifying factors}

Most of the interviewed women were married young women being pregnant for the first or second time. Consequently, the majority might desire further pregnancies, with enhanced risks of becoming HIV positive and of transmitting HIV to their children. "Child bearing is a very important event for every Zimbabwean. Yet the desire of the couple with HIV infection to have children need to be balanced with the possibility of having an infected baby who has a high risk of dying within the first 5 years of life" (MOHCW, 1999, p. 10).

The finding that $80.0 \%$ of the women were married and that no one was a widow, could be attributed to sustained polygamous practices in Zimbabwe (Goercke, 2004). "It is traditional practice in the Shona culture that if a woman's husband dies, then the deceased husband's brother inherits the widow" (Goercke, 2004, p. 17). The issues of polygamy and 'widow inheritance' imply that a woman in Zimbabwe is not only at risk of becoming HIV positive from her husband (and from his brother in the case of 'widow inheritance') but also from all these men's other wives/sexual partners and from all these women's sexual partners. This makes the prevention of vertical transmission of HIV a serious concern in Bulawayo, where the majority of the population are Shonas.

As all the women could communicate in English, and as many had reached secondary or even tertiary education levels, these women should be able to benefit from health education sessions about the prevention of vertical transmission of HIV. Despite their relatively high levels of education, only $30.0 \%$ $(n=15)$ were employed, implying that $70.0 \%(n=35)$ would be financially dependent on their husbands/partners. Such financial dependence could limit the women's negotiating powers with their partners concerning all aspects of preventing vertical transmission of HIV/AIDS including the use of condoms, getting the husband/partner tested for HIV, using anti-retroviral drugs and not breastfeeding the baby.

\subsection{Barriers to implementing effective health actions}

\subsubsection{Communication with husbands/partners about preventive MTCT actions}

The major barrier concerned the fact that $38.0 \%(n=19)$ of the women could not discuss the possibility of implementing preventive vertical transmission actions with their husbands/partners. In Zimbabwe men still pay a bridal prize, known as "lobola", comprising large sums of money or many heads of cattle, for each wife. "It is generally believed that when a man marries and pays lobola for a woman, he owns her" (Ndlovu, 1997, p. 20). In return for the financial investment in his wife, the husband expects her to obey his commands, work in the fields and bear a large number of children. The husband decides whether and when his wife could seek medical advice. As $70.0 \%(n=35)$ of the women interviewed in this study were unemployed, and as $80.0 \%$ $(n=40)$ were married, the majority of women were financially dependent on their husbands. Against this background information few women might use prevention of vertical transmission services, even if they knew that they were HIV positive. "In the developing societies of Southern Africa ... women face a greater risk of HIV infection than men because of diminished socio economic status which compromises their ability to choose safer and healthier life styles" (Van Rensburg et al., 2002, p. 29).

\subsubsection{Breastfeeding}

The knowledge that vertical transmission can occur through breastfeeding and the availability of free formula did not enable the women to refrain from breastfeeding. The reason given was the lack of money to pay for transport to collect the formula from the clinics at regular intervals. However, in a culture where every mother is expected to breastfeed her baby, artificial feeding will be regarded suspiciously by the woman's husband, family and community members, stigmatizing the woman and her baby as being HIV positive. Such stigmatization can cause the woman and her child(ren) to be abandoned by the husband/partner and to become social outcasts, making survival under harsh circumstances almost impossible.

\subsubsection{Mode of delivery}

Although $23(46.0 \%)$ of the interviewed women would prefer vaginal deliveries even if they were HIV positive, 27 $(54.0 \%)$ would prefer caesarian sections to reduce the possibility of vertical transmission of HIV/AIDS. Caesarian sections cannot be performed routinely on HIV positive women in Zimbabwe because of severe shortages of facil- 
ities and the expenses involved. In 2005, during the data collection phase of this study, the Government of Zimbabwe did not offer free obstetric nor pre- and postnatal services. A normal vaginal delivery costed $\mathrm{Z} \$ 1.5$ million (1.5 million Zimbabwean dollars which was the equivalent of US\$150.00); a traditional birth attendant charged $\mathrm{Z} \$ 0.5$ million (the equivalent of US\$50.00) while the cost of a caesarian section was Z\$4.0 million (US\$400.00). The costs of caesarian sections are beyond the financial reach of most Zimbabwean women because the average monthly wages for professional women in Zimbabwe were $\mathrm{Z} \$ 25$ million (US\$250.00) and for non-professional women Z\$10.00 million (US\$100.00) during 2005 (MOHCW, 2002).

\section{Limitations of the study}

The sample was limited to pregnant women attending one prenatal clinic who had booked to deliver their babies at one hospital in Bulawayo. These women had access to the prevention of vertical transmission services. Another sample of women who did not attend prenatal clinics and/or who preferred to deliver their babies at home, or with the assistance of a traditional birth attendant, might have yielded different data. A convenience sample of 50 women could not claim to represent the general population because not every element in the population had a chance of being included in the sample.

As the HIV status of participating women was unknown, no comparisons could be made between the actions and attitudes of HIV positive, HIV negative and HIV unknown status women.

Data were collected by conducting structured interviews only. More in-depth information about Bulawayo's women's lived experiences in implementing prevention of vertical transmission actions, could be obtained by conducting indepth individual interviews.

\section{Conclusions}

Almost one third of the women lacked knowledge about the prevention of vertical transmission and the role of breastfeeding. Even those women who knew about the prevention of vertical transmission could not use formula for their babies nor could they resort to caesarian sections. The availability of knowledge, antiretroviral drugs and formula feeds in Bulawayo did not save many babies from vertical transmission. This is the case because these women had to pay for pre- and postnatal care, exorbitant fees for caesarian sections, transport costs to obtain supplies of formula for their babies. Some women would breastfeed their babies even if they knew that they were HIV positive. Only $54.0 \%$ of the interviewed women were willing to be tested for HIV; only women who tested HIV positive can access prevention of vertical transmission services. As an unknown number of women in Bulawayo opt for the cheaper services of traditional birth attendants, they are denied access to prevention of vertical transmission services, offered only to HIV positive women who attend prenatal clinics and deliver their babies in hospital.

Unless men can be mobilised to participate in prevention of vertical transmission actions, few women will continue to use these services. A minority of women obtained their knowledge about the prevention of vertical transmission from health facilities, while the majority did so from the media.

\section{Recommendations}

The recommendations for enhancing the utilization of services preventing vertical transmission of HIV/AIDS in Bulawayo involve health care services; social, cultural, economic and gender issues.

Nurses should invest more time and effort in educating all women attending prenatal clinics about HIV/AIDS, including the prevention of vertical transmission of HIV/AIDS, and the role of breast feeding.

Health care policy changes could make vertical transmission prevention services more accessible to pregnant women in Bulawayo. Such policy changes include providing affordable (or free) pre- and postnatal as well as delivery services and subsidizing caesarian sections for HIV positive women.

Free formula feeds should be provided at venues other than hospitals and clinics, so that more women can access them. Village health workers and traditional birth attendants could help to distribute infant formula. Women selecting to deliver their babies at home and/or with traditional birth attendants should also have the option of using services for preventing vertical transmission of HIV/AIDS.

Zimbabawe's media should continue to broadcast information about HIV/AIDS, including the prevention of vertical transmission of HIV/AIDS, but these broadcasts should be continuously monitored by knowledgeable persons to ensure that the factual information remains correct.

Counseling should include men and VCT should be offered to a man and all his wives and sexual partners. Men should be encouraged to accompany their wives to prenatal clinics and attend health education sessions with their pregnant wives.

More women should be employed (a possibility judged by the women's high levels of education) so that they can be financially independent of their husbands and make independent decisions concerning the prevention of vertical transmission of HIV/AIDS.

Future research should strive to obtain information about women's lived experiences of implementing actions to prevent the vertical transmission of HIV/AIDS actions and should strive to distinguish among the actions of HIV positive, HIV negative and HIV status unknown groups of women in Zimbabwe.

HIV and AIDS are major public health problems throughout the world, particularly in developing countries with high 
fertility rates. "The concomitant high fertility and high HIV infection rates increase the risk of vertical transmission of HIV/AIDS. . . The importance of understanding the global effect of HIV infection among women is crucial to develop interventions to meet the challenges posed by this devastating disease on the health of vulnerable populations worldwide, and particularly in Africa" (Tabi and Frimpong, 2003, p. 249).

\section{Acknowledgments}

We would like to thank all the pregnant women who agreed to be interviewed and all the persons/institutions that granted permission for this study to be conducted.

\section{References}

Bang, A., 2000. Homecare for newborns. Southern Africa HIV/ AIDS Link, New Delhi.

Becker, M., 1974. The Health Belief Model and personal health behaviour. Health Education Monographs 2, 324-508.

Brink, H.I.L., 1999. Statistics for nurses. Academia, Pretoria.
Chivonivoni, C. 2006. Antenatal mothers' practices for preventing mother to child HIV/AIDS transmission. MA Cur dissertation. University of South Africa, Pretoria.

Goercke, B., 2004. The impact of traditional Shona beliefs on HIV/ AIDS intervention in Zimbabwe. Duquesne University, Pittsburg.

Ministry of Health and Child Welfare, 2002. Prevention of mother to child transmission of HIV in Zimbabwe. Training manual for health workers. Government Printer, Harare. MOHCW—see Ministry of Health and Child Welfare (of Zimbabwe).

Onega, L.L., 2000. Educational theories, models, and principles applied to community and public health nursing in Stanhope, M.S., \& Lancaster, J., 2000. Community and public health nursing. CV Mosby, St Louis. pp266-283.

Ndlovu, M., 1997. Violence against Women in the Midlands' Region: Strategies for action. Musasa Project, Harare.

Polit, D.F., Hungler, B.P., 1999. Nursing research principles and methods, 6th ed. JB Lippincott, Philadelphia.

Southern Africa HIV/AIDS Action, 2000. Southern Africa HIV/ AIDS Link, Worldwide, London.

Tabi, M.M., Frimpong, S., 2003. HIV infection of women in African countries. International Nursing Review 50 (4), 242-250.

Van Rensburg, D., Friedman, I., Ngwena, G., Pelser, A., Booysen, F., Adendorff, E., 2002. The dynamics of AIDS in South Africa. Centre for Health Systems Research and Development, Pretoria. 